\section{Quantifying Molecular Stiffness and Interaction with Lateral Force Microscopy}

\author{
Alfred John Weymouth, * Thomas Hofmann, Franz J. Giessibl \\ Universität Regensburg, Regensburg 93053, Germany. \\ *Corresponding author. E-mail: jay.weymouth@ur.de
}

The spatial resolution of atomic force microscopy (AFM) can be drastically increased by terminating the tip with a single CO molecule. However, the CO molecule is not stiff, and lateral forces, such as those around the sides of molecules, distort images. This issue begs a larger question of how AFM can probe structures that are laterally weak. Lateral force microscopy (LFM) can probe lateral stiffnesses that are not accessible to normal-force AFM, resulting in higher spatial resolution. With LFM, we determined the torsional spring constant of a COterminated tip molecule to be $0.24 \mathrm{~N} / \mathrm{m}$. This value is less than that of a surface molecule, and an example of a system whose stiffness is a product not only of bonding partners but also local environment. asymmetry.

We acquired a 3D dataset by collecting constant-height normal-force AFM images at various distances from the surface (21). To isolate the shortrange interaction, we subtracted the raw $\Delta f$ data from the $\Delta f$ signal above the bare $\mathrm{Cu}$ surface (14). The data were then deconvoluted and integrated twice along the $z$-direction (where $z$ denotes the distance to the surface) to yield the potential energy (21-23). The potential energy map of the short-range interaction at closest approach is shown in Fig. $1 B$.

A plot of the energy as a function of tip height above the surface $\mathrm{CO}$ molecule is given in Fig. 1C. Figure 1C also includes a fit of the energydistance curve to a Morse potential (24):
True atomic resolution was demonstrated for AFM more than 15 years ago, but only recently has it been possible to atomically engineer the tip apex $(1-4)$, a technique pioneered in the STM community $(5,6)$. Tip termination with a CO molecule has made studies of inter- and intramolecular bonding possible (7-11). The CO-terminated tip itself, however, has proven to be difficult to characterize, despite experimental and theoretical approaches $(12,13)$, in part because it is challenging to characterize lateral forces and stiffnesses with AFM. With any study of shortrange interactions, AFM requires that the long-range background interaction of the macroscopic tip and surface be subtracted (14). Although a full three-dimensional (3D) dataset can reconstruct the potential energy, lateral forces and stiffnesses are only accessible by taking a numerical derivative of experimentally determined energies (15), which results in substantial noise in the data.

In LFM, the tip is oscillated parallel to the surface $(16,17)$, and the recorded frequency shift $(\Delta f)$ is a direct measure of the lateral stiffness (18). The subtraction of long-range contributions, necessary in normalforce AFM (where the tip oscillates normal to the surface) to identify the short-range force components, is obsolete in LFM, rendering LFM particularly appealing for measurements in which the short-range interaction is the signal of interest (e.g., Ref. (19)). We have used both normalforce AFM and LFM to characterize a CO terminated tip and quantify parameters of a model incorporating torsional springs to account for the response of the $\mathrm{CO}$ molecules to lateral forces.

When the CO tip was first used to image pentacene, it was remarked that the image seemed to be distorted due to $\mathrm{CO}$ relaxation (7). Further experiments showed that the relaxation of the CO molecule on the tip was an important part of the tip-surface interaction (13). This relaxation is usually characterized as a torsional spring, with the CO bending around the metal atom to which it is bound (12).

We used a CO-terminated tip to probe a single CO molecule on $\mathrm{Cu}(111)$. $\mathrm{CO}$ on $\mathrm{Cu}(111)$ has been well-studied and the system offers a high degree of symmetry. The asymmetry in a normal-force AFM image of a CO with a CO-terminated tip (Fig. 1A) is the result of the slight asymmetry of the tip with respect to the surface, amplified by the bending of both tip and surface CO molecules. Images collected at further distances [e.g., those shown in Fig. S1 (20)] do not show this degree of

$$
E_{M}=E_{B}\left(-2 \exp \left[-\frac{r-\sigma}{\lambda}\right]+\exp \left[-2 \frac{r-\sigma}{\lambda}\right]\right)
$$

The distance $r$ describes the core-core distance between the two oxygen atoms. We comment on the equilibrium distance, $\sigma$, later. The bond energy, $E_{B}=8.4 \mathrm{meV}$, was determined by the energy minimum and the decay length, $\lambda=47$ p.m., was determined by a least-squares fit from the data further out from the equilibrium distance. For these longer distances, the interaction is attractive, and the molecules do not bend. At closer distances, we expect the repulsive interaction to lead to the CO molecules bending away from each other.

We then used a lateral force sensor (17), also with a CO-terminated tip, to probe a surface $\mathrm{CO}$ molecule. The mathematical description of the frequency shift and its relation to force remains the same: interaction with the surface changes the frequency of oscillation proportional to the force gradient, $k_{\mathrm{ts}}$, along the direction of oscillation. The effect of the cantilever oscillation is that we measured a weighted average, $\left\langle k_{\mathrm{ts}}\right\rangle$, over the cantilever's oscillation (25). The frequency shift was directly proportional to $\left\langle k_{\mathrm{ts}}\right\rangle$, via the spring constant of the cantilever, $k$, and its resonance frequency $f_{0}: \Delta f=\left\langle k_{\mathrm{ts}}>f_{0} /(2 k)\right.$.

The raw image of the CO molecule with LFM (Fig. 2A) revealed a circular shape with a highly localized depression in the middle. The high spatial resolution in this LFM image could not be reconstructed from data acquired with a normal-force AFM: Similar to the method of Ref (15), we can evaluate lateral stiffnesses via the potential energy from the normal-force AFM data (inset of Fig. 2A), but after the two numerical derivatives, the sharp localized depression is not reproduced. Although one solution to the noise caused by the derivatives would be to use a low-pass filter, this step would only decrease the spatial resolution. Thus, it was not feasible to reconstruct this sharp feature using only normal-force AFM data.

We next acquired constant-height LFM slices above the CO molecule. Figure 2C shows a line scan through the image at closest approach over the CO molecule in the direction of the tip oscillation. Because this line was in the same direction as the tip oscillation, it could be deconvoluted and integrated to yield lateral force in that direction. We repeated this process for several tip-sample distances. The colored graph in Fig. 
2D shows the lateral force over the CO molecule as a function of both lateral and vertical position. As we expect from the normal-force AFM data, the lateral force shows attractive interaction as the $\mathrm{CO}$ molecules approach each other, then repulsive interaction closer than the equilibrium distance. Assuming that the $\mathrm{CO}$ bends to relax, we would expect $\mathrm{CO}$ bending for all positions except when the lateral forces are equal to zero.

We calculated the lateral and vertical position where the lateral forces are equal to zero. These zero crossings were fit to a circle as shown in Fig. 2D. This result implies that there was a very weak angular dependence for the CO-CO interaction, which is another advantage of a COterminated tip. The zero crossings of the force are the locations of the energy minimum, and thus the radius of the circle, 385 p.m., is the equilibrium distance between the oxygen atoms, $\sigma$. Absolute height determination is also a challenge in scanning probe techniques. With the assumption that the interaction is not directionally dependent, $\sigma$ can be used to determine the absolute height of our tip above the surface CO molecule, as given in Fig. 2D.

We now consider the challenge of determining the relaxation of the $\mathrm{CO}$ molecule experimentally. In order to make this problem tractable, we can separate the total interaction into three components: 1 ) the interatomic interaction between the two oxygen atoms; 2) the CO relaxation of the tip molecule; and 3) the CO relaxation of the surface molecule. We propose the following model: 1) The interatomic interaction can be described by a Morse potential, which we have fully characterized with $E_{B}$, $\lambda$ and $\sigma ; 2$ ) The surface $\mathrm{CO}$ molecule responds as a torsional spring with a torsional spring constant of $\kappa_{\mathrm{S}}=150 \mathrm{zNm}$ and a moment arm of $l_{\mathrm{CO}}=$ 302 p.m. (26); 3) The tip CO molecule relaxes as a torsional spring with an unknown spring constant $\kappa_{\mathrm{T}}$ but with the same moment arm $l_{\mathrm{CO}}$. The moment arm was determined by a theoretical study which determined the core-core distance of the $\mathrm{Cu}$ surface atom to the $\mathrm{C}$ and $\mathrm{O}$ atoms (26). Helium scattering measurements have previously determined the energy of the frustrated translational mode to be $4 \mathrm{meV}$ (27). With the moment arm, these values can then be used to determine the torsional spring constant $\kappa_{\mathrm{S}}$ as described in (3).

A schematic of the distances in the model is shown in Fig. 3A. At every position of the final tip atom that does not relax $(x, z)$, the total energy of the system can be calculated as a function of the bending angle of the tip CO molecule $\theta_{\mathrm{T}}$ and of the surface CO molecule $\theta_{\mathrm{S}}$ :

$$
E=E_{M}(r)+\frac{1}{2} \kappa_{T} \theta_{T}^{2}+\frac{1}{2} \kappa_{S} \theta_{S}^{2}
$$

$r$ is the core-core distance between the oxygen atoms, which is a function of $x, z, \theta_{\mathrm{T}}$ and $\theta_{\mathrm{S}}$. For every tip position $(x, z)$ we minimize the energy with respect to $\theta_{\mathrm{T}}$ and $\theta_{\mathrm{S}}$, which thus yields the energy of the system at that position. We can differentiate this energy surface to yield the force and force gradient. The force gradient can then be convoluted with a semicircular weight function (to account for the cantilever motion) and multiplied by $f_{0} /(2 k)$ to explicitly determine $\Delta f$, which can be compared directly to our data.

A spectrum of LFM data in red points (Fig. 3B) was collected as a function of the vertical distance of the tip above the $\mathrm{CO}$ molecule. For a given value of $\kappa_{\mathrm{T}}$, we can use the model to generate an equivalent spectrum. We varied $\kappa_{\mathrm{T}}$ for a least-squares fit to the data. The best fit, shown by a blue solid line in Fig. 3B, yielded a value of $\kappa_{\mathrm{T}}=22 \mathrm{zNm}$. This value can be expressed as a linear spring constant $k_{\mathrm{T}}=\kappa_{\mathrm{T}} /\left(l_{\mathrm{CO}}\right)^{2}=0.24$ $\mathrm{N} / \mathrm{m}$. We can then simulate a full LFM image from this model, shown in Fig. 2B. The agreement is easier to appreciate comparing line scans, shown in Fig. 2C. Data and model output further from the surface [Fig. S2 (20)] also show excellent agreement.

Previous DFT-based calculationsof $k_{\mathrm{T}}$ ranged from 0.3 to $1.6 \mathrm{~N} / \mathrm{m}$, with previous experimental evidence pointing to a value lower than 0.5 $\mathrm{N} / \mathrm{m}$ (12). Our value of $0.24 \mathrm{~N} / \mathrm{m}$ agrees with this assessment, in particu- lar that this value is much lower than that of the surface CO molecule. This lower value is expected because the apex of the tip does not present a full surface to the CO molecule, but rather a fraction thereof. The following conclusion can be drawn, that the $\mathrm{CO}$ on the surface is laterally much stiffer and therefore a better probe. To increase the lateral stiffness of the tip, the CO molecule should be picked up on a blunt metal tip.

The LFM images have a strong signal that is much more spatially confined than the normal-force AFM images. Physically, the spatial resolution of frequency modulation AFM is limited by the lateral extent of the force gradient. Especially in the case of a CO-terminated tip, where lateral forces change rapidly over a sharp transition such as encountering an adsorbate, LFM increases the spatial resolution.

\section{References and Notes}

1. L. Gross, F. Mohn, N. Moll, P. Liljeroth, G. Meyer, The chemical structure of a molecule resolved by atomic force microscopy. Science 325, 1110-1114 (2009). doi:10.1126/science.1176210 Medline

2. F. Mohn, B. Schuler, L. Gross, G. Meyer, Different tips for high-resolution atomic force microscopy and scanning tunneling microscopy of single molecules. Appl. Phys. Lett. 102, 073109 (2013). doi:10.1063/1.4793200

3. J. Welker, F. J. Giessibl, Revealing the angular symmetry of chemical bonds by atomic force microscopy. Science 336, 444-449 (2012) doi:10.1126/science.1219850 Medline

4. J. Welker, A. J. Weymouth, F. J. Giessibl, The influence of chemical bonding configuration on atomic identification by force spectroscopy. ACS Nano 7, 7377-7382 (2013). doi:10.1021/nn403106v Medline

5. D. M. Eigler, C. P. Lutz, W. E. Rudge, An atomic switch realized with the scanning tunneling microscope. Nature 352, 600-603 (1991). doi:10.1038/352600a0

6. L. Bartels, G. Meyer, K.-H. Rieder, Controlled vertical manipulation of single CO molecules with the scanning tunneling microscope: A route to chemical contrast. Appl. Phys. Lett. 71, 213 (1997). doi:10.1063/1.119503

7. N. Moll, L. Gross, F. Mohn, A. Curioni, G. Meyer, The mechanisms underlying the enhanced resolution of atomic force microscopy with functionalized tips. New J. Phys. 12, 125020 (2010). doi:10.1088/13672630/12/12/125020

8. M. P. Boneschanscher, J. van der Lit, Z. Sun, I. Swart, P. Liljeroth, D. Vanmaekelbergh, Quantitative atomic resolution force imaging on epitaxial graphene with reactive and nonreactive AFM probes. ACS Nano 6, 10216 10221 (2012). doi:10.1021/nn3040155 Medline

9. J. Zhang, P. Chen, B. Yuan, W. Ji, Z. Cheng, X. Qiu, Real-space identification of intermolecular bonding with atomic force microscopy. Science 342, $611-$ 614 (2013). doi:10.1126/science.1242603 Medline

10. N. Pavliček, B. Fleury, M. Neu, J. Niedenführ, C. Herranz-Lancho, M. Ruben, J. Repp, Atomic force microscopy reveals bistable configurations of dibenzo[a,h]thianthrene and their interconversion pathway. Phys. Rev. Lett. 108, 086101 (2012). doi:10.1103/PhysRevLett.108.086101 Medline

11. F. Albrecht, M. Neu, C. Quest, I. Swart, J. Repp, Formation and characterization of a molecule-metal-molecule bridge in real space. J. Am. Chem. Soc. 135, 9200-9203 (2013). doi:10.1021/ja404084p Medline

12. L. Gross, F. Mohn, N. Moll, B. Schuler, A. Criado, E. Guitián, D. Peña, A. Gourdon, G. Meyer, Bond-order discrimination by atomic force microscopy. Science 337, 1326-1329 (2012). doi:10.1126/science.1225621 Medline

13. Z. Sun, M. P. Boneschanscher, I. Swart, D. Vanmaekelbergh, P. Liljeroth, Quantitative atomic force microscopy with carbon monoxide terminated tips. Phys. Rev. Lett. 106, 046104 (2011). doi:10.1103/PhysRevLett.106.046104 Medline

14. M. Ternes, C. González, C. P. Lutz, P. Hapala, F. J. Giessibl, P. Jelínek, A. J. Heinrich, Interplay of conductance, force, and structural change in metallic point contacts. Phys. Rev. Lett. 106, 016802 (2011). doi:10.1103/PhysRevLett.106.016802 Medline

15. M. Ternes, C. P. Lutz, C. F. Hirjibehedin, F. J. Giessibl, A. J. Heinrich, The force needed to move an atom on a surface. Science 319, 1066-1069 (2008). doi:10.1126/science.1150288 Medline

16. O. Pfeiffer, R. Bennewitz, A. Baratoff, E. Meyer, P. Grütter, Lateral-force measurements in dynamic force microscopy. Phys. Rev. B 65, 161403 (2002). doi:10.1103/PhysRevB.65.161403

17. F. J. Giessibl, M. Herz, J. Mannhart, Friction traced to the single atom. Proc. 
Natl. Acad. Sci. U.S.A. 99, 12006-12010 (2002). doi:10.1073/pnas.182160599 Medline

18. A. J. Weymouth, D. Meuer, P. Mutombo, T. Wutscher, M. Ondracek, P. Jelinek, F. J. Giessibl, Atomic structure affects the directional dependence of friction. Phys. Rev. Lett. 111, 126103 (2013). doi:10.1103/PhysRevLett.111.126103 Medline

19. D. Rugar, R. Budakian, H. J. Mamin, B. W. Chui, Single spin detection by magnetic resonance force microscopy. Nature 430, 329-332 (2004). doi:10.1038/nature02658 Medline

20. Materials and methods are available as supplementary materials on Science Online.

21. B. J. Albers, T. C. Schwendemann, M. Z. Baykara, N. Pilet, M. Liebmann, E. I. Altman, U. D. Schwarz, Three-dimensional imaging of short-range chemical forces with picometre resolution. Nat. Nanotechnol. 4, 307-310 (2009). doi:10.1038/nnano.2009.57 Medline

22. J. E. Sader, S. P. Jarvis, Accurate formulas for interaction force and energy in frequency modulation force spectroscopy. Appl. Phys. Lett. 84, 1801-1803 (2004). doi:10.1063/1.1667267

23. J. Welker, E. Illek, F. J. Giessibl, Analysis of force-deconvolution methods in frequency-modulation atomic force microscopy. Beilstein J. Nanotechnol. 3, 238-248 (2012). doi:10.3762/bjnano.3.27 Medline

24. P. Morse, Diatomic Molecules According to the Wave Mechanics. II. Vibrational Levels. Phys. Rev. 34, 57-64 (1929). doi:10.1103/PhysRev.34.57

25. F. J. Giessibl, Advances in atomic force microscopy. Rev. Mod. Phys. 75, 949-983 (2003). doi:10.1103/RevModPhys.75.949

26. M. Gajdoš, J. Hafner, $\mathrm{CO}$ adsorption on $\mathrm{Cu}(111)$ and $\mathrm{Cu}(001)$ surfaces: Improving site preference in DFT calculations. Surf. Sci. 590, 117-126 (2005). doi:10.1016/j.susc.2005.04.047

27. J. Braun, A. P. Graham, F. Hofmann, W. Silvestri, J. P. Toennies, G. Witte, A He-atom scattering study of the frustrated translational mode of CO chemisorbed on defects on copper surfaces. J. Chem. Phys. 105, 3258 (1996). doi:10.1063/1.471841

Acknowledgments: We thank D. Meuer for the LFM sensor construction, A.-K. Greitner for COMSOL modelling of the LFM sensor, and the DFG (GRK 1570) for financial support.

\section{Supplementary Materials}

www.sciencemag.org/content/science.1249502/DC1

Materials and Methods

Figs. S1 to S2

9 December 2013; accepted 23 January 2014

Published online 6 February 2014

10.1126/science.1249502 

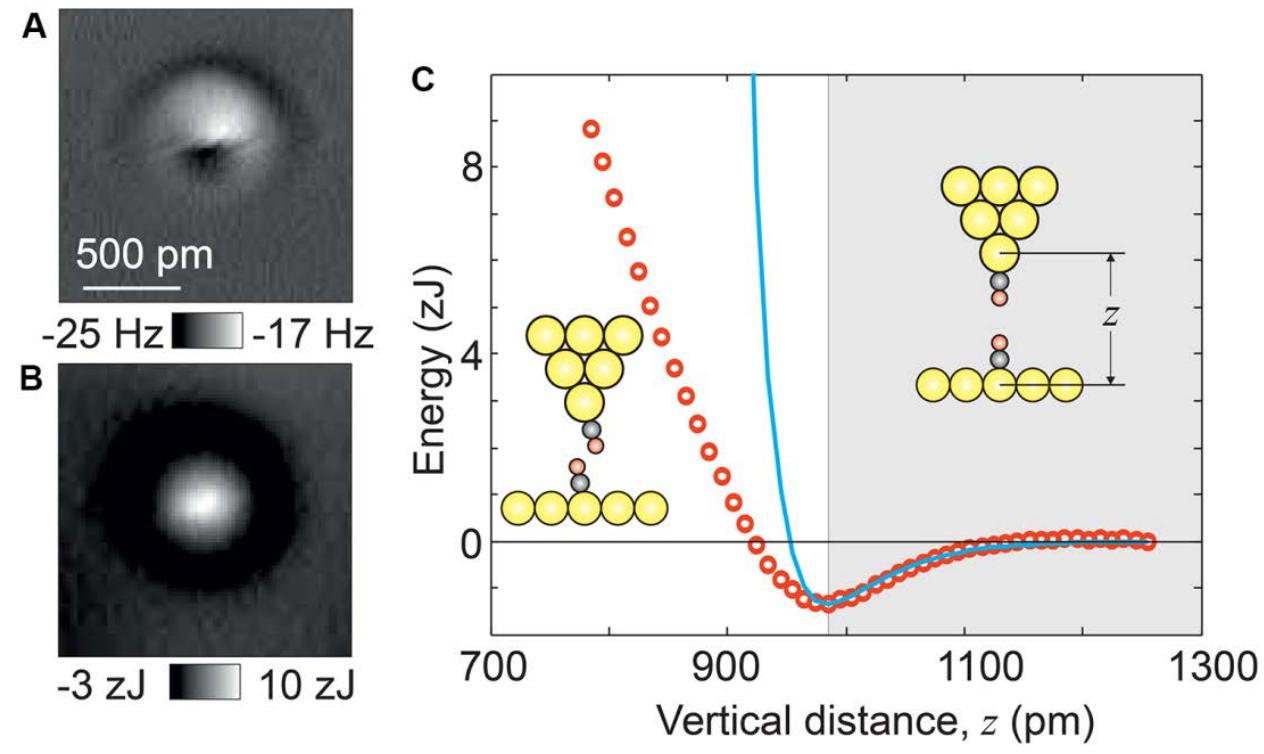

Fig. 1. AFM imagined with a CO-terminated tip. (A) Normal AFM data of a CO molecule with a CO-terminated tip. (B) Deconvolved energy image. (C) Energy as a function of vertical tip position when the tip is directly above the CO molecule. Red dots are data, blue curve is a fit of a Morse potential to the data.
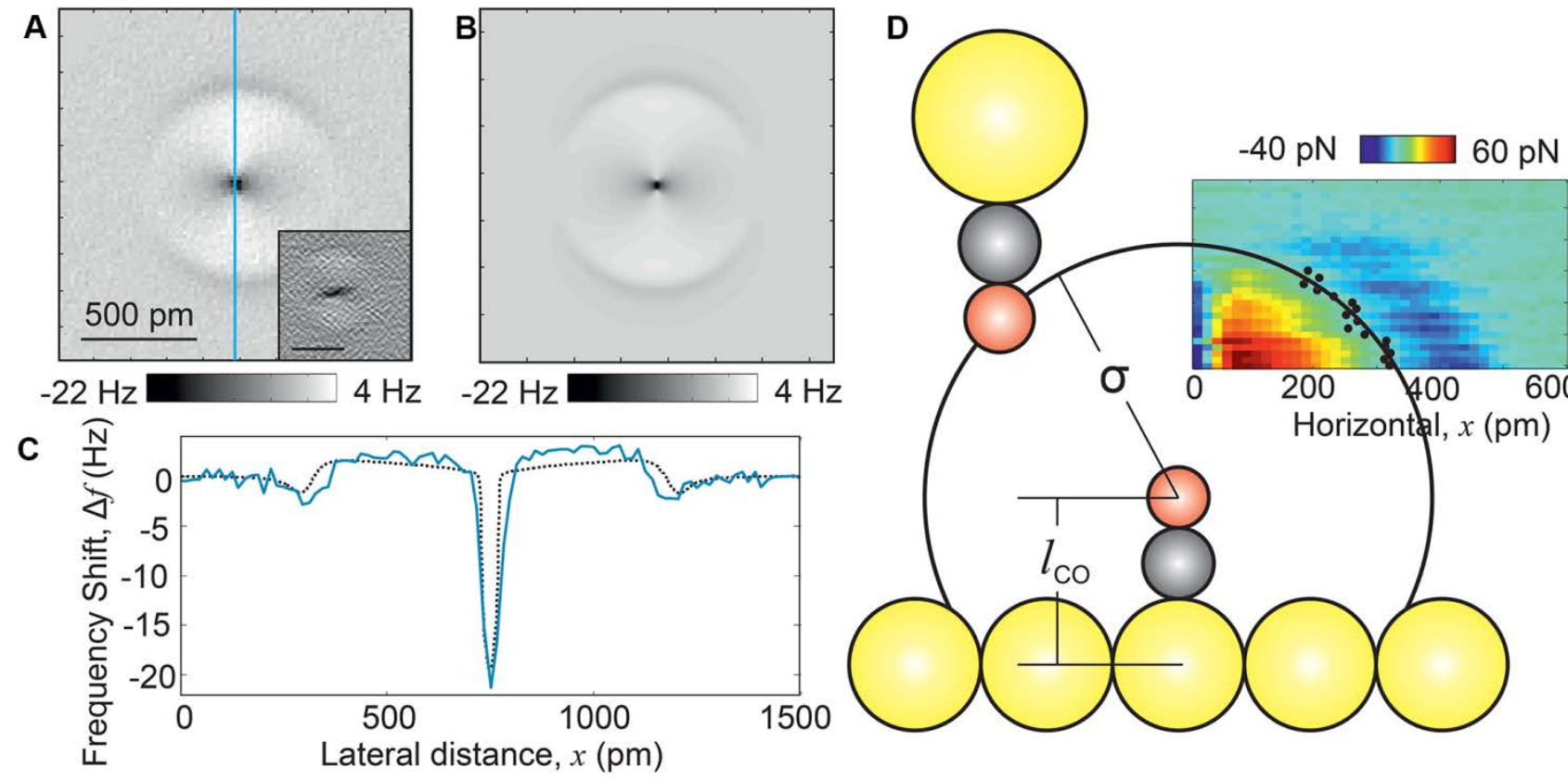

Fig. 2. LFM with a CO-terminated tip. (A) Raw data from a LFM sensor. Tip oscillation is in the same direction as the solid blue line. Inset: Lateral stiffness as evaluated from normal AFM data. Scale bar is also 500 p.m.(B) Model output with the same scale as (A). (C) A line scan through the center of the $\mathrm{CO}$ molecule in the direction of oscillation. Solid blue line is the data, dashed black line is model output. (D) The colored inset shows the lateral forces. Black points indicate where the lateral force is zero. These points lie on a circle whose radius defines the bond length between the oxygen atoms. 
A

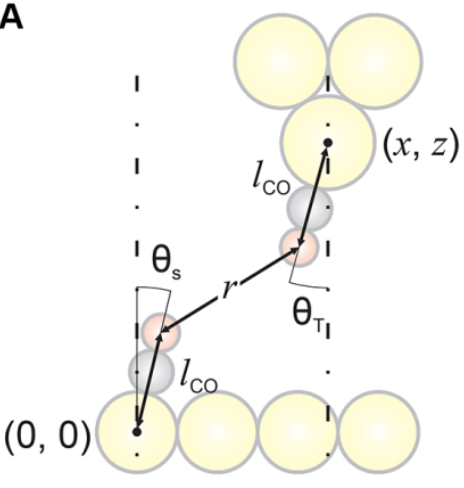

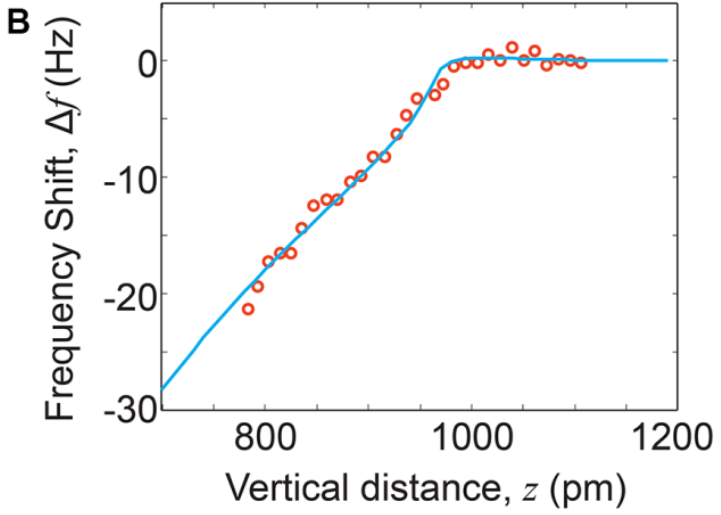

Fig. 3. Modeling the LFM process. (A) In our model, the final tip atom to which the $\mathrm{CO}$ is bound is set at $(x, z)$. The tip CO molecule is allowed to relax by an angle $\theta_{T}$, and the surface molecule to relax by an angle $\theta_{\mathrm{s}}$. (B) LFM data above the CO molecule as a function of vertical distance (z). Solid red points are experimental and the blue curve is the calculated output. 


\section{Science MIAAAS}

Quantifying Molecular Stiffness and Interaction with Lateral Force Microscopy

Alfred John Weymouth, Thomas Hofmann and Franz J. Giessibl (February 6, 2014)

published online February 6, 2014

Editor's Summary

This copy is for your personal, non-commercial use only.

Article Tools Visit the online version of this article to access the personalization and article tools:

http://science.sciencemag.org/content/early/2014/02/05/science.1249502

Permissions Obtain information about reproducing this article: http://www.sciencemag.org/about/permissions.dtl

Science (print ISSN 0036-8075; online ISSN 1095-9203) is published weekly, except the last week in December, by the American Association for the Advancement of Science, 1200 New York Avenue NW, Washington, DC 20005. Copyright 2016 by the American Association for the Advancement of Science; all rights reserved. The title Science is a registered trademark of AAAS. 